\title{
Analysis of Native Milk Oligosaccharides Directly from Thin-Layer Chromatography Plates by Matrix-Assisted Laser Desorption/ Ionization Orthogonal-Time-of-Flight Mass Spectrometry with a Glycerol Matrix
}

\author{
Klaus Dreisewerd, Stefanie Kölbl, and Jasna Peter-Katalinić \\ Institute of Medical Physics and Biophysics, Westfälische-Wilhelms Universität Münster, Münster, Germany
}

\author{
Stefan Berkenkamp \\ Sequenom GmbH, Hamburg, Germany
}

Gottfried Pohlentz

Institute of Medical Physics and Biophysics, Westfälische-Wilhelms Universität Münster, Münster, Germany

\begin{abstract}
We have recently presented a new method for direct coupling of high-performance thin-layer chromatography (HPTLC) with matrix-assisted laser desorption/ionization mass spectrometry (MALDI-MS), illustrated by the analysis of a complex ganglioside mixture (Dreisewerd et al., Anal. Chem. 2005, 77, 4098). In the current communication, an adaptation of this procedure to mixtures of native oligosaccharides from human and from elephant milk is described. The key features in this method are (1) glycerol as a liquid matrix, to provide a homogeneous wetting of the silica gel and a simple and fast MALDI preparation protocol, (2) an infrared (IR) laser for volume material ablation and particular soft desorption/ionization conditions, and (3) an orthogonal time-of-flight mass spectrometer for a high mass accuracy, independent of any irregularity of the silica gel surface. Chromatographic "mobility profiles" were determined by scanning the laser beam across the analyte bands. The current limit of detection for the MS analysis was determined to $\sim 10 \mathrm{pmol}$ of individual oligosaccharides spotted for chromatography. A liquid composite matrix, containing glycerol and the ultraviolet (UV-)MALDI matrix $\alpha$-cyano-4-hydroxycinnamic acid, allows a direct HPTLC-MALDI-MS analysis with a 337 nm-UV laser as well. Compared to the IR-MALDI mode, the analytical sensitivity in UV-MALDI was found to be lower by one order of magnitude, whereas unspecific analyte ion fragmentation as well as adduct formation was found to be more extensive. (J Am Soc Mass Spectrom 2006, 17, 139-150) (c) 2006 American Society for Mass Spectrometry
\end{abstract}

$\mathrm{T}$ The combination of robust and low-cost, but rather low-resolving high-performance thin-layer chromatography (HPTLC) with mass spectrometry (MS) systems for the precise analysis of samples of particular interest offers an attractive hybrid technique. HPTLC is frequently used for separation and partial characterization of neutral and acidic oligosaccharides from mixtures $[1,2]$. The low resolving power of the method, however, does not usually permit differentiation of species of similar structure and "mobility", and the chromatograms serve mostly for visualization and as a finger-print. Both electrospray ionization (ESI

Published online January 10, 2006

Address reprint requests to Dr. Klaus Dreisewerd, Institute of Medical Physics and Biophysics, University of Münster, Robert Koch Strasse 31, D-48149 Münster, Germany. E-mail: dreisew@uni-muenster.de
[3]) and matrix-assisted laser desorption/ionization (MALDI [4]) mass spectrometry can be applied for analysis of oligosaccharides from solutions $[5,6]$. Either based on a high mass accuracy alone in the standard MS mode [7] or by tandem MS analysis [8], these techniques can in many cases provide an unambiguous identification of the samples. However, for analysis of oligosaccharide mixtures derived from a single core structure by extension with single monosaccharide units, e.g., fucose, it can often not be differentiated whether an ion detected by $\mathrm{MS}^{1}$ is a molecular ion species or an in-source cleavage product. By separation of the mixtures by HPTLC into defined bands, before the MS analysis from distinct positions, this valuable information can be obtained. Similarly, liquid chromatography (LC-) ESI- or LC-MALDI-MS can provide this information. These latter methods constitute the standard tech- 
niques for the analysis of oligosaccharide mixtures and allow for sensitivity in the low pmol range. However, samples need, in these cases, either to be analyzed on-line or to be fractionated before the analysis. One advantage of the HPTLC-separation is thus that it provides a planar separation from which the MALDI-MS analysis can be performed any time later.

Different methods for direct coupling of HPTLC and MALDI-MS have been described previously; overviews are provided in references [9-10]. Because of the routine use of HPTLC for primary characterization of glycolipids, the direct HPTLC-MS analysis of these compounds has so far found particular interest [11-13]. A particular advantage of direct HPTLC-MALDI-MS is that it allows a simple switching from one spot of interest to another. Technically, it is also easier to realize it than on-line HPTLC-ESI-MS [14, 15]. Besides, requirements on the purity of the samples are generally less strict for MALDI-MS analysis. However, in most investigations reported so far, the direct HPTLC-MALDI-MS analysis has been associated with a relatively low mass resolution and accuracy [13]. With the "axial" time-offlight (TOF) instruments employed in these studies, these limitations can directly be attributed to the desorption from the coarse, nonconducting silica gel surfaces: broad initial ion velocity distributions lead to poorly resolved ion signals.

Mass spectrometers in which the desorption/ionization process is basically decoupled from the mass determination were recently shown to provide advanced solutions for these problems. In particular, Fourier-transform ion cyclotron resonance (FTICR-) [11, 16] ion trap- [17], and orthogonal (o-) TOF instruments [12] are suitable platforms to achieve this goal. A further advantage of these instruments is that they allow the use of ion sources with elevated background pressure which can stabilize labile ions by collisional cooling $[16,18]$. As a positive side effect, these sources also come along with reduced requirements on the vacuum system and do thus allow the use of even large quantities of relatively volatile matrices without degrading the instrumental performance. Almost all HPTLC-MALDI-MS studies have so far been performed with solid MALDI matrices, which are either spotted or sprayed upon the silica gel [9]. These matrices, generally successful for ultraviolet (UV-)MALDI, have the inherent disadvantage in that the extraction of analyte compounds from the silica gel and a proper cocrystallization with the analyte/silica gel system is mandatory for good analytical results. Only a small layer of material about tens of nanometers in depth is, moreover, desorbed per laser pulse in UV-MALDI [19]. In contrast, significant volumes of material about one to ten $\mu \mathrm{m}$ in depth are ablated per by an infrared (IR-)MALDI [12, 22].

We have recently demonstrated the feasibility of direct HPTLC-MALDI-o-TOF-MS with an Er:YAG IRlaser and glycerol as a liquid matrix for the analysis of singly sialylated GM3 gangliosides [12]. Technically, this approach is in a close analogy to earlier studies in which fast-atom bombardment (FAB) was used to generate molecular ions of glycoconjugates directly from TLC plates [20, 21]. In comparison to FAB, IR-MALDI, in combination with the glycerol matrix, provides considerably softer desorption/ionization conditions; this has been demonstrated for various classes of biomolecules [22] including complex carbohydrates [23].

More than 130 different oligosaccharide structures have so far been described for human milk [2, 24]. The exact composition varies between individuals and in the time-course of lactation [25]. The total lactosederived oligosaccharide content in human milk is about 5 to $8 \mathrm{~g} / 1$ (compared with 55 to $70 \mathrm{~g} / 1$ of free lactose) [24]. Cow milk, in contrast, like that from most other mammalians, contains only traces of oligosaccharides [24]. This complex oligosaccharide content in human milk has been previously recognized for its role in the immune system of the infant [24]. Whereas human milk was considered to be unique in terms of its complex oligosaccharide content for some time, more recently elephant milk has been shown to represent another exception [2]. Previous studies, performed in our laboratory employing FAB high-energy collision-induced (CID) tandem MS on permethylated oligosaccharides, have revealed that in elephant milk only oligosaccharides of the neo-lacto (Type II) series are expressed (Pohlentz et al., unpublished data). This and other MS studies using high-energy CID [26, 27] showed that human milk, in contrast, contains both the lacto (Type I) and neo-lacto isoforms. Moreover, in elephant milk galactosylated and fucosylated isoforms are found with about equal abundances (data not shown) whereas human milk contains exclusively the fucosylated type.

In the present report, the direct HPTLC-IR-MALDIo-TOF-MS analysis of two chromatographic fractions of native oligosaccharides from human milk (HM) and one from elephant milk (EM) is described. Glycerol was employed as a liquid matrix. In addition, a liquid composite matrix for direct HPTLC-UV-MALDI-MS was also tested.

\section{Experimental}

\section{Chemicals}

Glycerol (p.a.), $\alpha$-cyano-4-hydroxycinnamic acid (CHCA), 3-aminoquinoline, ammonium phosphate, the calibrant peptides, bradykinin fragment 1-7, and melittin, and all solvents were from Sigma-Aldrich (Deisenhoven, Germany) and were used as delivered. Purified Fuc-LNT (specified purity $>95 \%$ ) was from Dextra Laboratories, Reading, UK.

\section{Composition of Oligosaccharides}

Milk oligosaccharide building monosaccharides are D-glucose (Glc), D-galactose (Gal), D-N-acetylglucosamine (GlcNAc), L-fucose (Fuc), and sialic acid [D-N- 
acetylneuraminic acid (NeuAc)]. The core structures consist of $\mathrm{N}$-acetyllactosamine $(\mathrm{Gal} \beta 1-(3,4) \mathrm{GlcNAc})$ units attached to the 3 and/or 6 position of the preceding galactose and carry lactose at their reducing ends. Thus, a large number of linear or branched cores can be formed. Additional substitution with fucose, galactose, and sialic acid moieties at different positions of the core contribute further to the complexity of milk oligosaccharide structures $[24,28]$.

\section{Preparation of Milk Samples}

Human milk was pooled from a breast-feeding individual donor. Fractions of human milk oligosaccharides were prepared essentially as described previously [29]. Briefly, the milk was centrifuged for $1 \mathrm{~h}$ at $2500 \times g$ $\left(4{ }^{\circ} \mathrm{C}\right)$ and the fat layer was removed. Proteins and most of the lactose were precipitated by adjusting the aqueous phase to $50 \%$ acetone. Separation of the remaining lactose and acidic oligosaccharides was achieved by chromatography on Sephadex G 10 and Sephadex DEAE 25 (Pharmacia, Uppsala, Sweden), respectively. Thirteen fractions were collected from the final size exclusion chromatographic step of the neutral oligosaccharides on Fractogel TSK HW 40S (Merck, Darmstadt, Germany) and the fractions "HM-7" and "HM-10" were used in the present study.

A milk sample from an individual Asian elephant (Elephas maximus) was kindly provided by Dr. Michael Flügger (Tierpark Hagenbeck, Hamburg, Germany). Oligosaccharide fractions were obtained by a modified procedure of Kunz et al. [2]. Milk samples were defatted by centrifugation as described above. Proteins were precipitated by adding ethanol at $4{ }^{\circ} \mathrm{C}$ to a final concentration of $66 \%$. The final crude oligosaccharide fractions 2 (large acidic), 3 (small acidic), 4 (large neutral), 5 (small neutral), and 6 (lactose) were obtained by size exclusion chromatography on Sephadex G 25 (Pharmacia, Uppsala, Sweden). Fraction "EM-4" was analyzed in the present report.

The obtained amount of oligosaccharides in all fractions was determined by weighing the lyophilized fractions. Desired concentrations were produced by adding according volumes of water.

\section{High-Performance Thin-Layer Chromatography}

The oligosaccharide solutions were separated on glassbacked silica gel 60 precoated HPTLC plates (no. 5633; Merck, Darmstadt, Germany; size $10 \times 10 \mathrm{~cm}$; thickness of the silica gel layer, $0.2 \mathrm{~mm}$ ). HPTLC plates were used as supplied by the manufacturer. Oligosaccharides were separated by developing the plate twice in nbutanol/acetic acid/water (110:45:45, vol/vol/vol) in a saturated chamber with intermediate drying. MSanalysis was performed from unstained lanes. For MS analysis, the HPTLC plates were cut into pieces of up to $30 \times 50 \mathrm{~mm}$ in size. Three parallel lanes of the samples, developed on the same plate, were stained with orcinol for reference [30]. The part of the plate containing these three lanes was cut, dipped into $0.3 \%$ (wt/vol) orcinol in $3 \mathrm{M} \mathrm{H}_{2} \mathrm{SO}_{4}$ for $10 \mathrm{~s}$, and subsequently transferred onto a preheated heating plate $\left(110^{\circ} \mathrm{C}\right)$ until the appearance of pinkish-violet spots.

\section{Composite UV-MALDI Matrix}

The stock solution of the composite glycerol/CHCA/3aminoquinoline matrix was prepared according to Cramer and Corless [31]. Briefly, CHCA, 3-aminoquinoline, and glycerol were mixed together in a microcentrifuge tube in a 1:4:6-ratio by weight. The mixture was sonicated until a clear yellow solution was obtained. Finally, ammonium phosphate was added to a concentration of $25 \mathrm{mM}$.

\section{Orthogonal-TOF-Mass Spectrometer}

The o-TOF-mass spectrometer is a modified prototype, similar to the one described by Loboda et al. [18]. By default, this instrument is equipped with a $\mathrm{N}_{2} \mathrm{UV}$-laser emitting $3 \mathrm{~ns}$ long pulses at a wavelength of $337 \mathrm{~nm}$ and a repetition rate of $30 \mathrm{~Hz}$. The $\mathrm{N}_{2} \mathrm{UV}$-beam is delivered via a fiber optical system, producing a spot size on the sample of $\sim 200 \times 230 \mu \mathrm{m}^{2}$. In our modified version, an IR-MALDI port has been added. Two similar Er:YAGlasers, (1): Bioscope, BiOptics Laser Systems AG, Berlin, Germany; (2): Speser, Spektrum Laser $\mathrm{GmbH}$, Berlin) were used for IR-MALDI. Both lasers emit pulses of $\sim 100 \mathrm{~ns}$ duration at a wavelength of $2.94 \mu \mathrm{m}$ and a repetition rate of $2 \mathrm{~Hz}$, but produced slightly different laser spots. A 2:1-telescope was used to expand the IR laser beams and an $\mathrm{f}=200 \mathrm{~mm}$ lens to focus them onto the sample under an angle of $45^{\circ}$. The focal spot size of the Bioscope laser was $\sim 150 \times 200 \mu \mathrm{m}^{2}$, as derived by optical inspection of the ablated zone on the silica gel. The second laser produced a principal spot of $100 \times 200$ $\mu \mathrm{m}^{2}$, which was accompanied by satellite spot, about $150 \mu \mathrm{m}$ apart in the chromatographic direction. The satellite spot contained a tenth to a few ten percent of the energy in the principal spot, as judged from the ablation on the silica and was also $\sim 100 \times 200 \mu \mathrm{m}^{2}$ in size. In all these cases, the smaller diameter corresponds to the direction of the chromatographic separation. Samples are observed with a CCD-camera of $10 \mu \mathrm{m}$ optical resolution. A custom-made, milled-out stainless steel sample plate was used to accommodate TLC cut-outs of up to $50 \times 30 \mathrm{~mm}^{2}$ in size.

Ions are generated in an elevated-pressure ion source filled with nitrogen $\left(p \sim 1-5 \times 10^{-1} \mathrm{mbar}\right)$ and are accelerated by a low extraction field of $\sim 25 \mathrm{~V} / \mathrm{mm}$ into a quadrupole ion guide. The quadrupole is filled with $\mathrm{N}_{2}$ at a pressure of $\sim 10^{-3} \mathrm{mbar}$. The ion transmission of the quadrupole was set to an $\mathrm{m} / \mathrm{z}$ window of $\sim 400-$ 4000. After extraction through a differentially pumped region, ions enter the low-pressure part of the instrument $\left(p \sim 1 \times 10^{-7}\right.$ mbar) where they are accelerated in orthogonal direction with respect to their original 
movement. Analysis was exclusively performed in the positive ion mode, with the TOF-pusher voltage set to $20 \mathrm{kV}$. Calibration of the instrument was achieved with a two-point calibration using molecular ions of bradykinin fragment 1-7 and melittin desorbed from a regular glycerol preparation. For acquisition of the IR-MALDI mass spectra, $\sim 100$ single laser pulses were typically applied. The laser fluence was adjusted to a value of about $10,000 \mathrm{~J} / \mathrm{m}^{2}$. Since at this fluence value, the 200 $\mu$ m-thick silica gel was typically removed after 20 to 30 single laser shots, the sample plate was moved to allow 4 to 5 positions on a horizontal line next to each other to be sampled. For acquisition of the UV-MALDI-mass spectra, $\sim 7500$ laser pulses were applied on a few ten different positions. Mass spectra were processed using the MoverZ3 software (version 2001.02.13, Genomic Solutions, Ann Arbor, MI).

\section{MALDI Preparation Protocols}

The direct HPTLC-MALDI-MS analyses were performed from unstained bands. Positions of the chromatographically separated bands were determined from the orcinol-stained reference lanes. For IR-MALDI, a vertical strip of glycerol-wetted silica gel was produced by applying a series of 0.2 to $0.4 \mu \mathrm{L}$ drops of pure glycerol next to each other. The glycerol was rapidly soaked up by the silica gel and diffused laterally, producing a uniformly wetted, $\sim 2$ to $3 \mathrm{~mm}$ wide strip. The wetted silica gel areas can easily be differentiated from the nonwetted by their whitish appearance. The composite UV-MALDI matrix was prepared in a similar way.

\section{Results and Discussion}

The three orcinol-stained reference lanes from a HPTLC separation of the two human and the elephant milk fractions are displayed in Figure 1. The numbers next to the analyte bands indicate the positions from which mass spectra (Figures 2, 3, 4, 5, and 6) were acquired. Positions below the center of the application zone, indicated by a dashed line, are denoted by negative numbers. For the HM-10 lane, the center-to-center distance between two neighboring irradiated positions was $\sim 300 \mu \mathrm{m}$, for the two other samples it was $\sim 400$ $\mu \mathrm{m}$. The HM-10 fraction was separated into two major bands (Figure 1a). These are expected to contain LNT and Fuc-LNT, respectively, as the major components, as determined by the FAB-(CID)-MS analysis of the unseparated fractions (data not shown). A third minor band at lower Rf value, expected to contain the doubly fucosylated LNT species, is only faintly visible in the reference chromatogram. Neither the chromatography nor the $\mathrm{MS}^{1}$ analysis allow to differentiate between isomeric structures of LNT and neo-lacto-N-tetraose (n-LNT), which may be both present in human milk; for simplicity, human milk oligosaccharides will, therefore, in the following be referred in the form of one of the

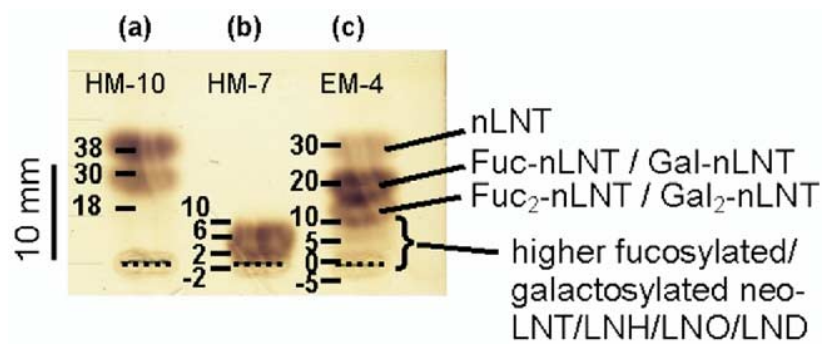

Figure 1. Orcinol-stained HPTLC chromatograms of human (HM) and elephant milk (EM) fractions. (a) HM-10; (b) HM-7; (c) EM-4. The horizontal lines at the bottom of the chromatograms indicate the approximate position at which samples were applied to the HPTLC plate. The figures next to the lanes indicate the laser positions at which the mass spectra, displayed in Figures 2-6, were acquired. All mass spectra were acquired from unstained lanes, which were developed in parallel on the same HPTLC plate. The center-to-center distance between two adjacent laser positions were $\sim 300 \mu \mathrm{m}$ for the analysis of HM-10 and $\sim 400 \mu \mathrm{m}$ for the experiments with the HM-7 and EM-4 samples. The assignments indicate the expected oligosaccharide species in the analyte bands for the EM-4 sample. Only fucosylated LNT but no Gal-LNT is expressed in human milk. Moreover, in human milk the core unit may contain both LNT and nLNT. Amounts of $6 \mu \mathrm{g}$ of HM-10, 5 $\mu \mathrm{g}$ of HM-7, and $\sim 10 \mu \mathrm{g}$ of total EM-4 oligosaccharides, respectively, were applied for HPTLC.

type I series isomers. The HM-7 fraction (Figure 1b), containing more complex oligosaccharides, was not resolved in discrete HPTLC bands. However, species of different size can be expected to be present within the optically unresolved wide area. Because the EM-4 elephant milk oligosaccharide sample was only roughly size-fractionated by the foregoing size exclusion chromatography, this fraction expectedly generated the most complex chromatogram. EM oligosaccharides are separated into four major bands (Figure 1c). Of these, the three upper ones are expected to contain nLNT and nLNT extended by a single or by two sugar residues, fucose or galactose, respectively. The structures of these compounds have been confirmed previously by FABhigh-energy CID-MS of the permethylated sample (data not shown). A series of larger, more complex oligosaccharides is distributed at low Rf values.

In Figure 2a-c, three mass spectra acquired from Positions 38, 30, and 18 of an unstained HM-10 lane of human milk are displayed. An amount of $0.6 \mu \mathrm{g}$ of milk oligosaccharides was applied for the separation; this is 10 times less than that used for the orcinol-stained reference lane. The three mass spectra were chosen to represent the two major and one minor analyte band, as shown in Figure 1a. The major ion species, detected at $\mathrm{m} / \mathrm{z} 730.24$ in the analyte band with the highest $\mathrm{Rf}$ value at Position 38 can straightforwardly be assigned to the sodiated $[\mathrm{M}+\mathrm{Na}]^{+}$molecular ion of lacto-N-tetraose (LNT, calculated $\mathrm{m} / \mathrm{z}$ 730.24; Figure 2a). The base peak, detected at $m / z, 876.30$ in the middle analyte band at Position 30 can be assigned to a singly fucosylated LNT (calculated $\mathrm{m} / \mathrm{z}, 876.30$; Figure 2b). Human milk is known to contain five different isomers of Fuc-LNT, differing in the binding site of the fucose residue and in 

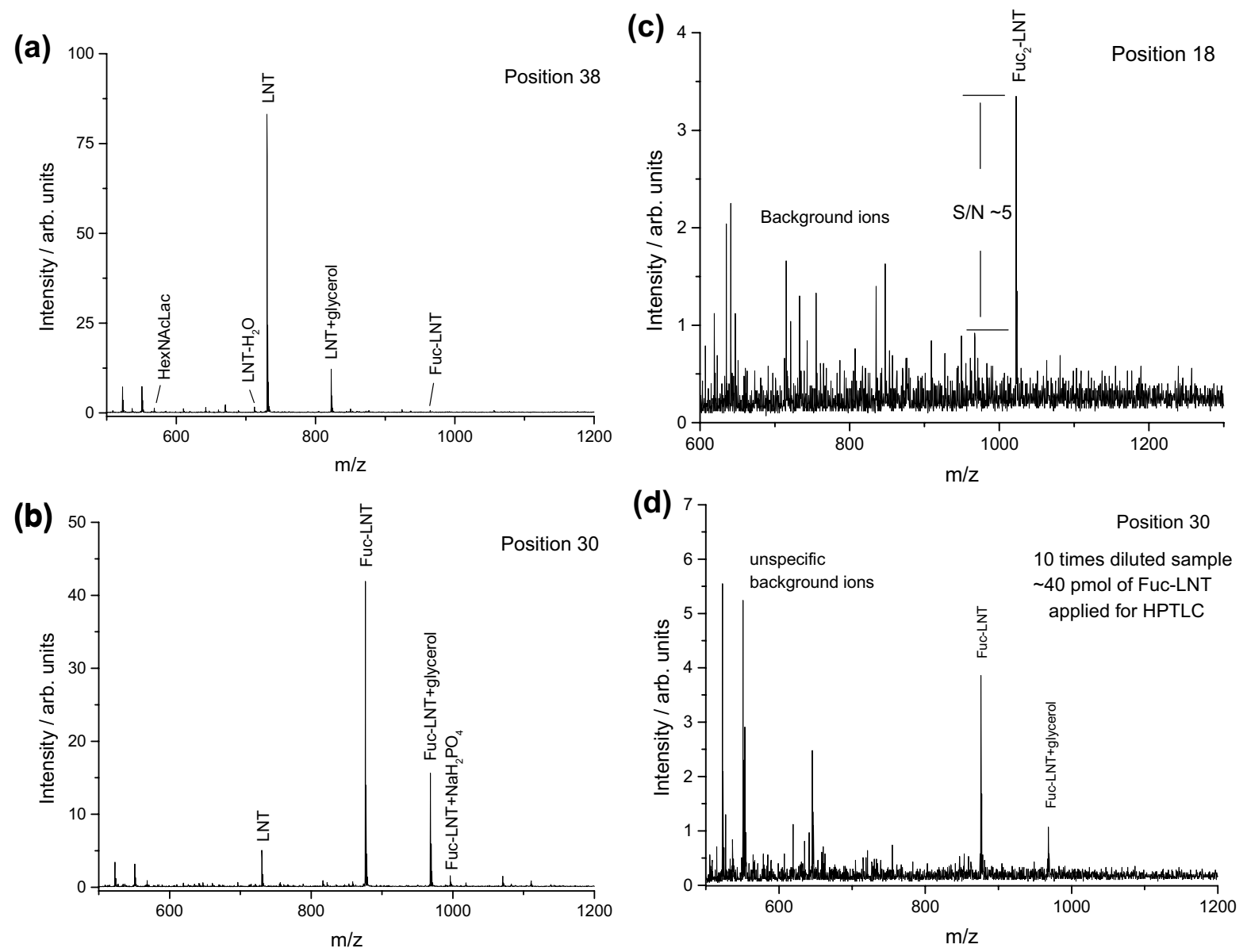

Figure 2. Direct HPTLC-IR-MALDI-o-TOF mass spectra of human milk fraction HM-10, recorded from different lateral positions on the chromatographic lanes, (a) at Position 38, (b) at Position 30, (c) at Position 18 (refer to Figure 1a for the assignment of the irradiated positions). An amount of $0.6 \mu \mathrm{g}$ of HM-10 oligosaccharides was, in this case, applied for HPTLC, ten times less than used for the reference chromatogram in Figure 1a. (d) Mass spectrum acquired from Position 30 of a 10 times more diluted sample, displaying the approximate limit of detection. Here, $\sim 60 \mathrm{ng}$ of total oligosaccharide sample was applied for HPTLC corresponding to $\sim 40$ pmol of Fuc-LNT.

the binding type [24]. These isomers cannot be differentiated from the $\mathrm{MS}^{1}$ spectrum. In the minor analyte band with the lowest Rf value a difucosylated LNT ion at $m / z 1022.37$ is present as the major component. All oligosaccharide species were detected as singly sodiated $[\mathrm{M}+\mathrm{Na}]^{+}$ions as the base peaks. The FWHM mass resolution was about 10,000 in all measurements.

In addition to the singly sodiated species, ions formed by adduct formation with glycerol matrix were also detected. Sizable intensities for these adduct compounds were only observed for LNT and Fuc-LNT but not for the more extended oligosaccharide species (see Figure $2 \mathrm{c}$ and below for the HM-7 and EM-4 fractions). A second series of low abundant adduct ion signals was generated by adduct formation with $\mathrm{NaH}_{2} \mathrm{PO}_{4}$ and $\mathrm{Na}_{2} \mathrm{HPO}_{4}$ (see Figure $2 \mathrm{~b}$ and below for the HM-7 fraction). The origin of the phosphate is not yet known; it is likely to be present in the original milk fraction. The parameters which might determine or influence the adduct formation, inclusive of that for unspecific dimers, will be investigated in more detail in successive studies.

The LNT signal, obtained from the middle HPTLC analyte band (Figure $2 \mathrm{~b}$ ) at about $10 \%$ intensity of the molecular Fuc-LNT signal, could originate from an insufficient chromatographic separation, or might be caused by an in-source cleavage of the fucose residue from the fucosylated precursor. In a control experiment with purified Fuc-LNT (purity $>95 \%$ as specified by the supplier), some LNT was in fact detected. A decrease of the laser fluence to values closer to the threshold fluence can be applied to reduce the degree of LNT formation by about an order of magnitude, but the acquisition times are then extended accordingly. A decrease of the acceleration potential in the ion source was also found to reduce the in-source fragmentation, but adduct formation and unspecific oligomerization of the oligosaccharide was then enhanced.

The limit of detection of the method in its present form is illustrated by the doubly fucosylated LNT ion 
(a)
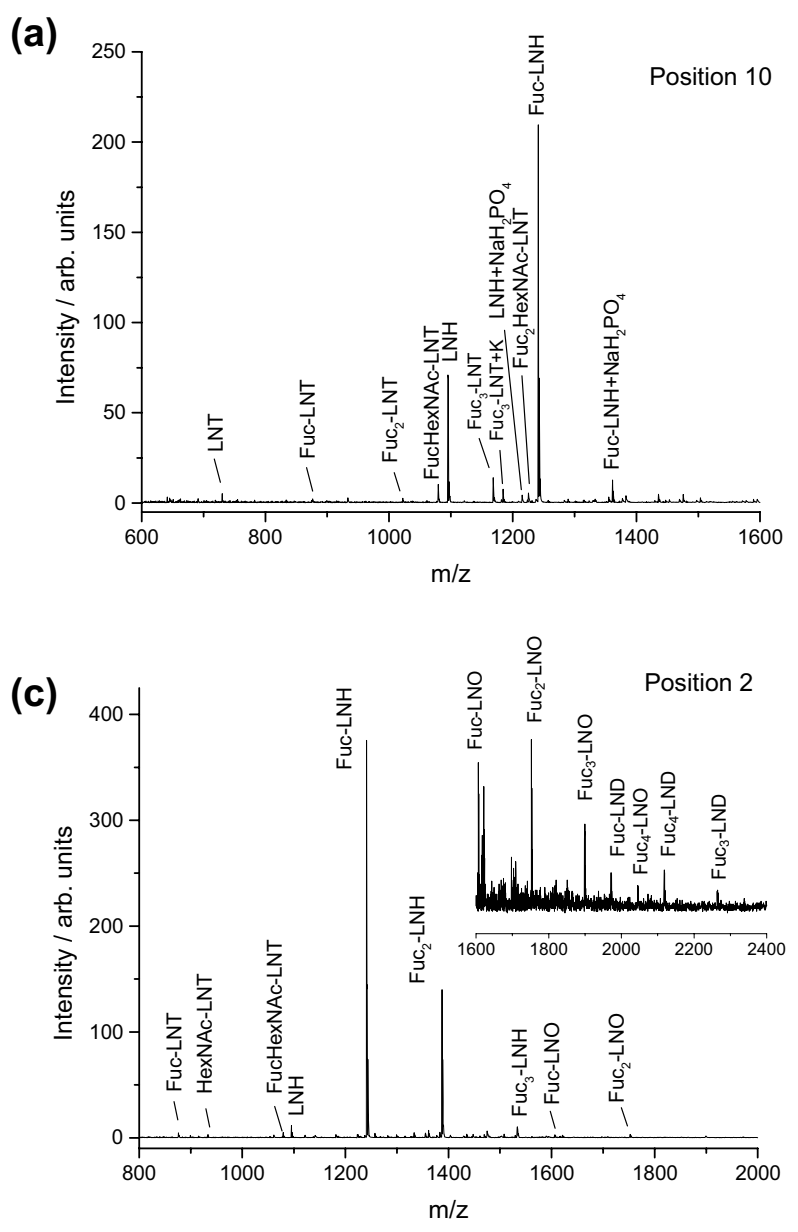

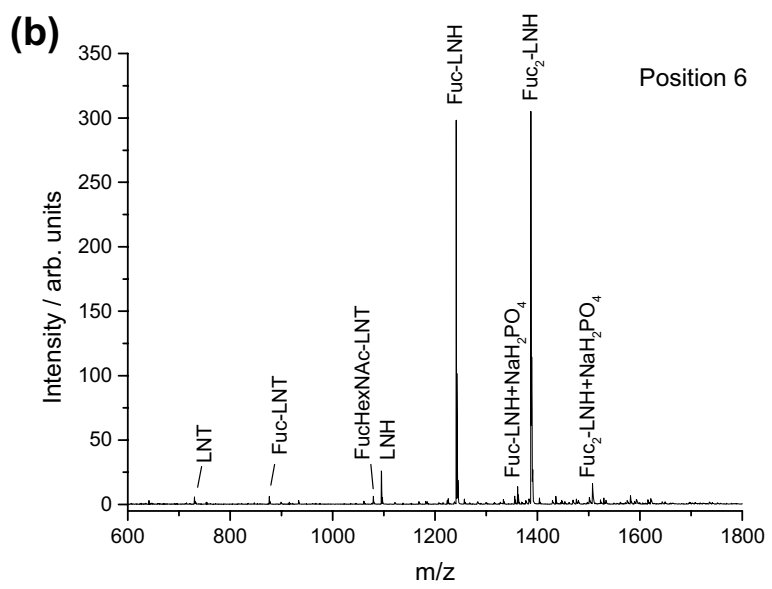

(d)

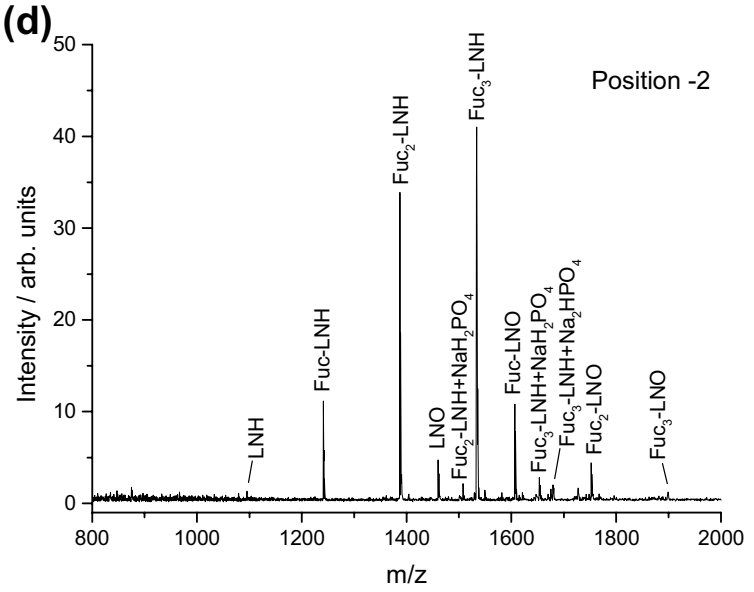

Figure 3. Direct HPTLC-IR-MALDI-o-TOF mass spectra of human milk fraction 7, recorded from different lateral positions on the chromatographic lanes, (a) at Position 10, (b) at Position 6, (c) at Position 2, (d) at Position -2 (refer to Figure $1 \mathrm{~b}$ for the assignment). Five $\mu$ g of HM-7 oligosaccharides were applied for HPTLC.

signal, generated from the minor analyte band at Position 18 (Figure 2c). Since the exact quantity of this species in the initial sample is not known, a dilution series was performed. The two major HM-10 signals, LNT and Fuc-LNT, representing about half of the oligosaccharide content of the HM-10 sample each, were still unambiguously detected from a 10 times more diluted sample, in which about $30 \mathrm{ng}$ of each species were contained, corresponding to about 40 pmol. A mass spectrum acquired from the middle analyte band is displayed in Figure 2d. Like for all of the mass spectra displayed above, a total of 100 single laser pulses only were applied. The LNT ion signal-tonoise ratio was about five (Figure 2d). A 10-fold further diluted sample did not deliver interpretable results anymore. The detection limit in the mass range below $1000 \mathrm{u}$ is apparently mostly determined by the background from the silica gel-matrix system. The limit of detection under the present experimental conditions is, therefore, about $10 \mathrm{pmol}$ of an individual oligosaccharide species applied to HPTLC.

A list of all molecular species detected by direct
HPTLC-IR-MALDI-MS at intermediate to high ion signal intensities from the HM-7 and HM-10 lanes is given in Table $1 \mathrm{a}$ and $\mathrm{b}$ along with their proposed identity and calculated $m / z$ values. All $m / z$ values are given for the sodiated quasimolecular ion or for adducts derived from this ion. The experimental and calculated mass values are determined to be within $20 \mathrm{ppm}$ for all species. An accuracy in the low ppm range should be achievable if instrumental and evaluation parameters are slightly adjusted [18, 32]. Besides for the isoform distinction, an unambiguous identification of the oligosaccharide species composition can be achieved.

As expected, in the HM-7 fraction oligosaccharide species with considerably higher complexity were detected. As examples, four mass spectra from Positions 10, 6, 2, and -2 of an unstained lane of HM-7 are shown in Figure 3 (cf. Figure $1 \mathrm{~b}$ for the reference chromatogram). Fucosylated lacto-Nhexaose (Fuc-LNH, $m / z$ 1241.44 $[\mathrm{M}+\mathrm{Na}]^{+}$) was the most abundant ion species in the mass spectrum recorded from Position 10 at a high Rf value (Figure $3 a$ ). In addition, the ion corresponding to the $\mathrm{LNH}$ core unit and a trifucosylated LNT species were found at higher abundances at $m / z 1095.38$ 

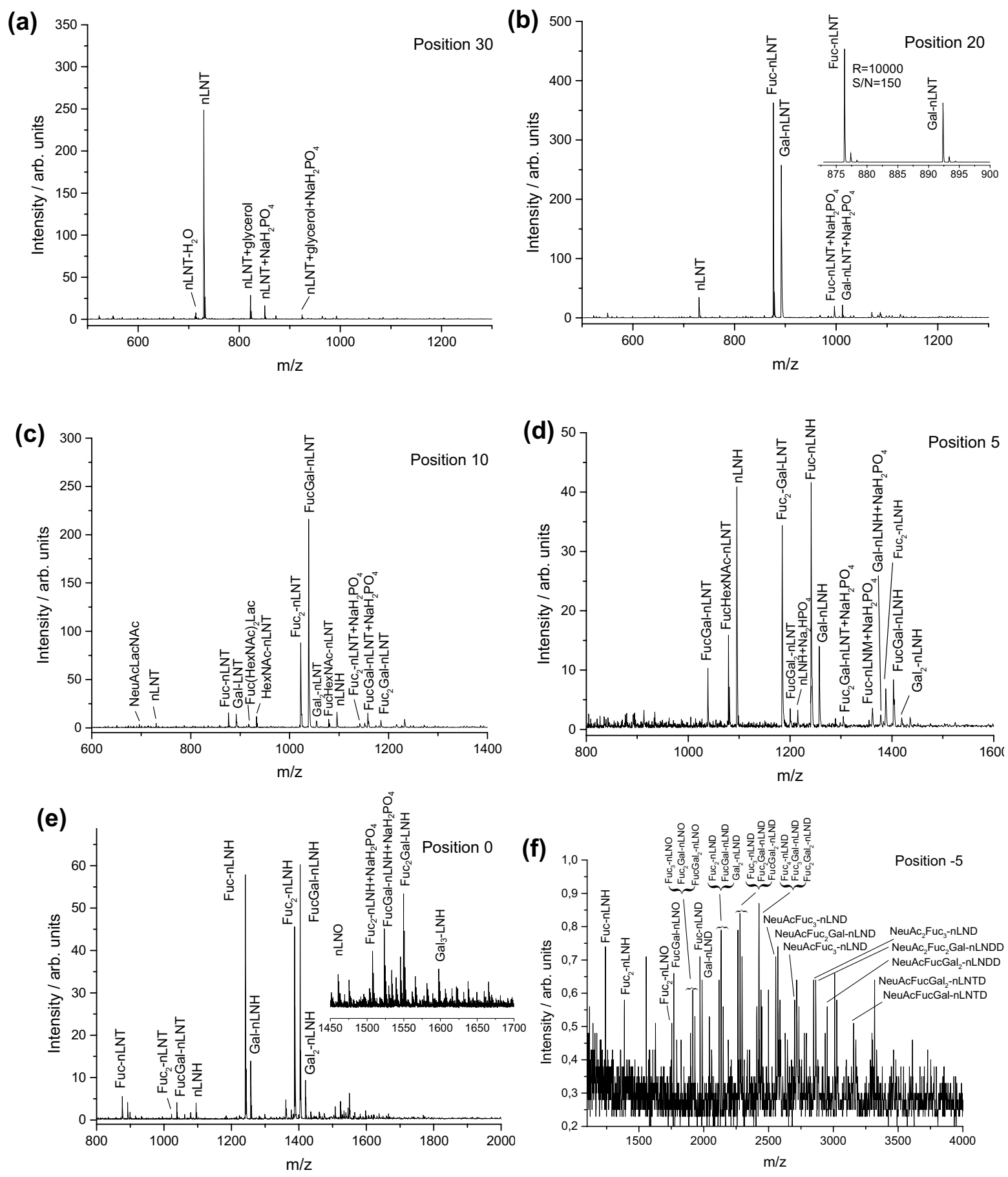

Figure 4. Direct HPTLC-IR-MALDI-o-TOF mass spectra of elephant milk fraction 4, recorded from different lateral positions on the chromatographic lanes, (a) at Position 30, (b) at Position 20, (c) at Position 10, (d) at Position 5, (e) at Position 0, (f) at Position -5 (refer to Figure 1c for the assignment). An amount of $\sim 10 \mu \mathrm{g}$ of EM-4 oligosaccharides was applied for HPTLC.

and 1168.42, respectively. Moreover, FucGlcNAcLNT and $\mathrm{Fuc}_{2}$ GlcNAc-LNT can be unambiguously correlated with their $\mathrm{m} / \mathrm{z}$ values at 1079.39 and 1215.33 , respectively. The most intense ion signals in the mass spectrum recorded from Position 6 were the singly and doubly fucosylated LNH form (Figure $3 \mathrm{~b}$ and Table $1 \mathrm{~b}$ ). Finally, mass spectra recorded close to the HPTLC application zone reveal those species which have hardly migrated. In the inset of Figure 3c, representing a mass spectrum recorded from Position 2, multiply fucosylated lacto-N-octaose (LNO) and lacto-N-decaose (LND) species with molecular weights up to $\sim 2200 \mathrm{u}$ are shown. As an intriguing feature of the approach, different major and minor oligosaccharides species with substantially varying abundance and molecular size were detectable in this spectrum. In Figure 3d, finally, the MS analysis of not migrated components is displayed, where $[\mathrm{M}+\mathrm{Na}]^{+}$ions of di- and 

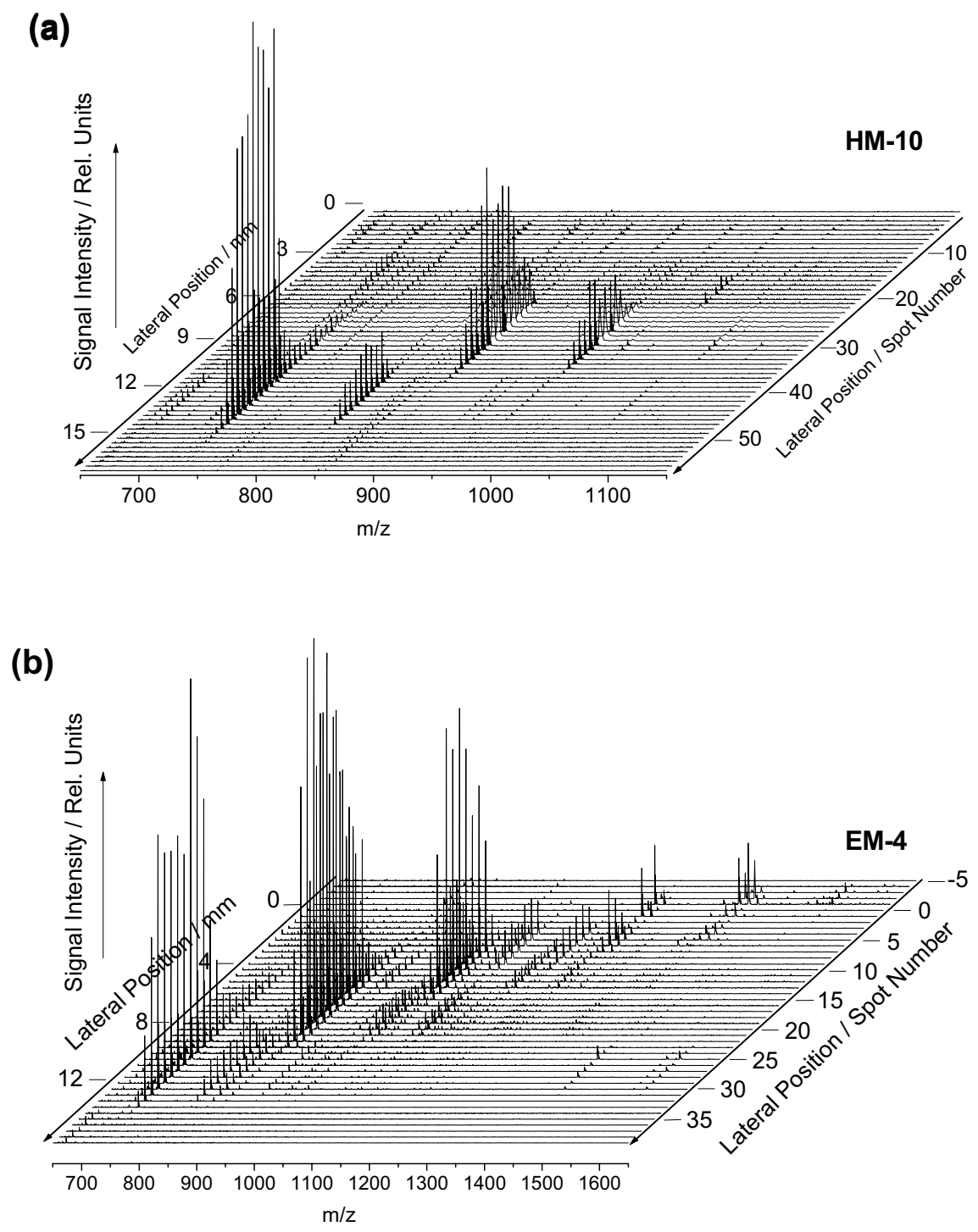

Figure 5. "2-D"-HPTLC-MS plot of (a) human milk fraction HM-10 and (b) elephant milk fraction EM-4 showing all recorded MS spectra as traces. The center-to-center distance between two irradiated positions is $\sim 300 \mu \mathrm{m}$ for the HM and $\sim 400 \mu \mathrm{m}$ for the EM sample.

trifucosylated LNH and LNO dominate. In agreement with the chromatogram (Figure 1b), a low overall ion signal intensity is found at this position.

Six direct HPTLC-MALDI mass spectra, acquired from the HPTLC lane of the EM-4 elephant milk fraction, are displayed in Figure $4 \mathrm{a}-\mathrm{f}$. The high complexity of the sample, already indicated by the reference chromatogram (Figure 1c), is corroborated by the mass spectra. In Table 1c all detected ion species with sizable intensity, along with their proposed identity and the $m / z$ values as calculated for the [M + $\mathrm{Na}]^{+}$ion are summarized. Aside from ions of very low abundance close to the detection limit experimental and calculated mass values agree well within $50 \mathrm{ppm}$ for all species. Since all experimental values for the EM sample are systematically somewhat too low in the second digit, a slight calibration offset is believed to account for this lower accuracy, compared with the $20 \mathrm{ppm}$ determined above for the two human milk samples. In Figure 4a, the mass spectrum recorded from the analyte band corresponding to the highest $\mathrm{Rf}$ values at Position 30 is presented; this band contains exclusively nLNT $(m / z, 730.29)$ with sizable abundance. In the second analyte band at Position 20, fucosylated and galactosylated nLNT species are dominating, yielding sodiated molecular ions at $\mathrm{m} / \mathrm{z}$ values of 876.33 and 892.33 , respectively (Figure $4 \mathrm{~b}$ ). The third in the reference chromatogram, clearly discernable analyte band contains $\mathrm{Fuc}_{2}-\mathrm{nLNT}$, FucGal-nLNT, and $\mathrm{Gal}_{2}$-nLNT as three major species. In Figure 4c the mass spectrum recorded from Position 10 on this band is displayed, showing the doubly fucosylated/galactosylated LNT ions as the base peaks. The dominant ion signals in the mass spectrum, recorded at Position 5, are assigned to nLNTderived oligosaccharides extended by three fucose 


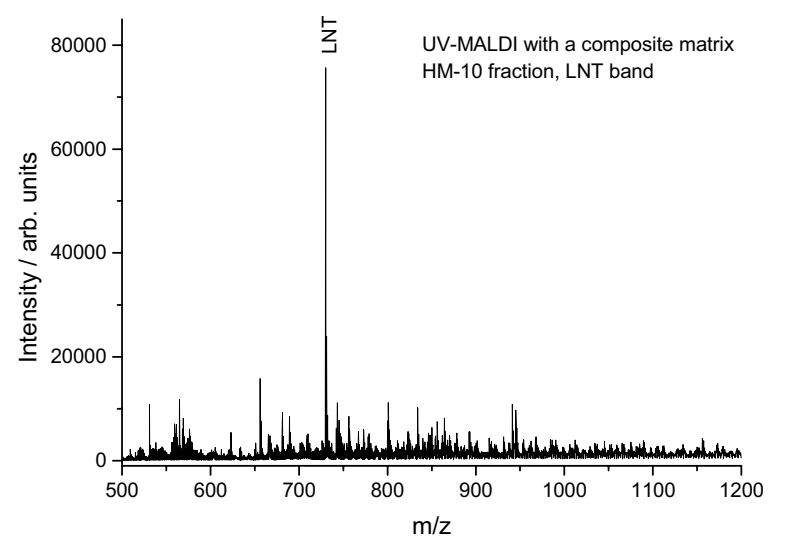

Figure 6. Direct HTPLC-MALDI-o-TOF mass spectrum of a human milk fraction HM-10 recorded from the upper analyte band with a UV laser using the liquid composite matrix. An amount of $0.6 \mu \mathrm{g}$ of HM-10 oligosaccharides was applied for HPTLC. The mass spectrum represents the sum of $\sim 7500$ laser pulses.

and/or galactose residues, as well as fucosylated and/or galactosylated nLNH-derived ions (Figure $4 d)$. In addition to fucose- and galactose-derived oligosaccharides, further species are detected at low Rf values, which by their molecular weight must be expected to contain $\mathrm{N}$-acetylhexosamine (HexNAc), e.g., at Positions 10 and 5. Like for the HM-7 sample, the most complex oligosaccharides are again found within or close to the sample application zone. In Figure 4e, the mass spectrum recorded from Position 0 is displayed, where one and two times fucosylated/ galactosylated $\mathrm{nLNH}$ ions are the main species. In addition, triply fucosylated/galactosylated $\mathrm{nLNH}$ was also found but at low abundance (see inset in Figure 4e). Finally, the most complex oligosaccharides with molecular weights exceeding $3000 \mathrm{u}$ are detected in the mass spectrum recorded from Position -5 , at the bottom limit of the application zone, where the $\mathrm{m} / \mathrm{z}$ values detected are assigned to the neo-lactoN-decaose (nLND) and -octaose (nLNO)-derived singly and multiply fucosylated/galactosylated ion species in the high mass range. The mass spectrum also reveals the presence of acidic $\mathrm{N}$-acetylneuraminic (NeuAc) acid containing oligosaccharides of even larger sizes which had apparently not been separated by the foregoing size exclusion chromatography.

The high complexity of the oligosaccharide composition in the elephant milk fraction is also illustrated by a "2-D" HPTLC-MS plot, displaying traces of all 39 mass spectra, which were recorded from the EM-lane. This reconstructed "MS image" is displayed in Figure 5b, showing the $m / z$ range between 600 and 1700. The MS image which was obtained from the HM-10 fraction with less complex composition is shown in Figure 5a. Because the HM-10 fraction could be separated into distinct analyte bands containing only one major analyte compound each, the "lateral resolution" of the MS-imaging can be at best illustrated by this latter plot. For the species with highest $R_{f}$ values, LNT, a width of $3 \mathrm{~mm}$ (FWHM) is revealed from Figure 5a, which corresponds well to the optically determined width of the orcinol-stained analyte band (Figure 1a).

\section{Composite Matrix}

A liquid composite matrix containing a highly-concentrated UV matrix component dissolved in glycerol can potentially allow an application of the here described method under use of more common UVMALDI lasers. Liquid composite matrices of this type have first been described by Sze et al. [33], and later refined by Cramer and Corless [31]. A direct HPTLCUV-MALDI mass spectrum, which was acquired from the upper LNT-containing band of the HM-10 chromatogram by using a glycerol/CHCA containing composite matrix, is displayed in Figure 6. An amount of $0.6 \mu \mathrm{g}$ of the HM-10 fraction was applied for HPTLC and about 7500 laser shots were accumulated from different neighboring positions. Generally, a decline in signal intensity was observed from one irradiated position after a few 100 laser shots were applied. Even after 1000 shots, an ablation of the silica gel was, however, not discernible with a highresolution microscope. Compared with the IRMALDI mass spectra (cf. Figure 2c), however, a substantially increased background is found. A comparison with a mass spectrum, obtained from a blank area of the developed HPTLC plate, reveals that most of this background is chemical noise related to both the MALDI matrix and silica gel derived cluster ions. A similarly high background has been observed in a very recent HPTLC-UV-MALDI-o-TOF-MS study on gangliosides in which sinapinic acid has been used as a solid UV-matrix [32]. Under the present experimental conditions, the limit of detection for direct HPTLC-UV-MALDI-MS with the composite matrix was about an order of magnitude lower than for IR-MALDI-MS with the pure glycerol matrix and about 100 pmol for the HM-10 oligosaccharides.

\section{Conclusions}

In line with our previous study on gangliosides [12], IR-MALDI-o-TOF-MS with a glycerol matrix provides an easy means of analyzing oligosaccharides directly from HPTLC plates. An advantage of the method compared with standard LC-ESI- or LCMALDI-MS is that it provides a planar separation from which the analysis can then be performed. Moreover, the sample preparation is straightforward and the use of the o-TOF-instrument allows a high accuracy in the mass determination independent of the morphology of the silica gel surface. An extension of the method for structural analysis by CID-MS, 
Table 1. Oligosaccharides detected in the human and elephant milk fractions by direct HPTLC-IR-MALDI-o-TOF-MS: (a) HM-10, (b) HM-7, (c) EM-4

\begin{tabular}{lcr}
\hline (a) & & \\
Oligosaccharide species & $m / z$ found for the $[\mathrm{M}+\mathrm{Na}]^{+}$ion & 568.18 \\
\hline \hline GIcNAc-Lac & 568.17 & 730.24 \\
LNT & 730.24 & 876.30 \\
Fuc-LNT & 876.29 & 1022.35 \\
Fuc $_{2}$-LNT & 1022.37 & $\mathrm{Na}]^{+}$ion \\
\hline
\end{tabular}

(b)

Oligosaccharide species

$m / z$ found for the $[\mathrm{M}+\mathrm{Na}]^{+}$ion

$m / z$ calculated for the $[\mathrm{M}+\mathrm{Na}]^{+}$ion

\begin{tabular}{|c|c|c|}
\hline LNT & 730.24 & 730.24 \\
\hline Fuc-LNT & 876.30 & 876.30 \\
\hline GlcNAc-LNT & 933.32 & 933.32 \\
\hline $\mathrm{Fuc}_{2}$-LNT & 1022.36 & 1022.35 \\
\hline FucGIcNAc-LNT & 1079.38 & 1079.38 \\
\hline LNH & 1095.37 & 1095.37 \\
\hline $\mathrm{Fuc}_{3}$-LNT & 1168.42 & 1168.41 \\
\hline $\mathrm{Fuc}_{2} \mathrm{GlcNAc} \mathrm{LNT}$ & 1225.44 & 1225.43 \\
\hline Fuc-LNH & 1241.43 & 1241.43 \\
\hline $\mathrm{Fuc}_{2}-\mathrm{LNH}$ & 1387.48 & 1387.49 \\
\hline $\mathrm{Fuc}_{3}-\mathrm{LNH}$ & 1533.54 & 1533.54 \\
\hline Fuc-LNO & 1606.56 & 1606.56 \\
\hline $\mathrm{Fuc}_{4}-\mathrm{LNH}$ & 1679.60 & 1679.60 \\
\hline $\mathrm{Fuc}_{2}-\mathrm{LNO}$ & 1752.61 & 1752.62 \\
\hline $\mathrm{Fuc}_{3}$-LNO & 1898.67 & 1898.68 \\
\hline Fuc-LND & 1971.67 & 1971.69 \\
\hline $\mathrm{Fuc}_{4}-\mathrm{LNO}$ & 2044.73 & 2044.73 \\
\hline $\mathrm{Fuc}_{2}$-LND & 2117.77 & 2117.75 \\
\hline $\mathrm{Fuc}_{3}$-LND & 2263.84 & 2263.81 \\
\hline
\end{tabular}

(c)

Oligosaccharide species

$m / z$ found for the $[\mathrm{M}+\mathrm{Na}]^{+}$ion

$m / z$ calculated for the $[\mathrm{M}+\mathrm{Na}]^{+}$ion

\begin{tabular}{|c|c|c|}
\hline $\mathrm{nLNT}$ & 730.26 & 730.24 \\
\hline Fuc-nLNT & 876.32 & 876.30 \\
\hline Gal-nLNT & 892.31 & 892.29 \\
\hline Fuc $(\mathrm{HexNAc})_{2}$ Lac & 917.36 & 917.32 \\
\hline HexNAc-nLNT & 933.34 & 933.32 \\
\hline $\mathrm{Fuc}_{2}-\mathrm{nLNT}$ & 1022.38 & 1022.35 \\
\hline FucGal-nLNT & 1038.36 & 1038.35 \\
\hline $\mathrm{Gal}_{2}-\mathrm{nLNT}$ & 1054.37 & 1054.34 \\
\hline FucHexNAc-nLNT & 1079.39 & 1079.38 \\
\hline $\mathrm{nLNH}$ & 1095.38 & 1095.37 \\
\hline $\mathrm{Fuc}_{2} \mathrm{Gal}-\mathrm{nLNT}$ & 1184.42 & 1184.41 \\
\hline Fuc-nLNH & 1241.44 & 1241.43 \\
\hline Gal-nLNH & 1257.44 & 1257.42 \\
\hline $\mathrm{Fuc}_{2}-\mathrm{nLNH}$ & 1387.52 & 1387.49 \\
\hline FucGal-nLNH & 1403.52 & 1403.48 \\
\hline $\mathrm{Gal}_{2}-\mathrm{nLNH}$ & 1419.53 & 1419.48 \\
\hline nLNO & 1460.54 & 1460.50 \\
\hline $\mathrm{Fuc}_{3}-\mathrm{nLNH}$ & 1533.58 & 1533.54 \\
\hline $\mathrm{Fuc}_{2} \mathrm{Gal}-\mathrm{nLNH}$ & 1549.57 & 1549.54 \\
\hline FucGal $_{2}-$ nLNH & 1565.56 & 1565.53 \\
\hline $\mathrm{Gal}_{3}-\mathrm{nLNH}$ & 1581.58 & 1581.53 \\
\hline Fuc-nLNO & 1606.60 & 1606.56 \\
\hline Gal-nLNO & 1622.59 & 1622.56 \\
\hline $\mathrm{Fuc}_{3} \mathrm{Gal}-\mathrm{nLNH}$ & 1695.63 & 1695.60 \\
\hline $\mathrm{Fuc}_{2} \mathrm{GaI}_{2}-\mathrm{nLNH}$ & 1711.64 & 1711.59 \\
\hline FucGal $_{3}-\mathrm{nLNH}$ & 1727.64 & 1727.59 \\
\hline $\mathrm{Fuc}_{2}-\mathrm{nLNO}$ & 1752.66 & 1752.62 \\
\hline FucGal-nLNO & 1768.67 & 1768.61 \\
\hline $\mathrm{Fuc}_{3}-\mathrm{nLNO}$ & 1898.73 & 1898.68 \\
\hline $\mathrm{Fuc}_{2} \mathrm{Gal}-\mathrm{nLNO}$ & 1914.71 & 1914.67 \\
\hline
\end{tabular}


Table 1. (continued)

\begin{tabular}{|c|c|c|}
\hline $\begin{array}{l}\text { (c) } \\
\text { Oligosaccharide species }\end{array}$ & $m / z$ found for the $[\mathrm{M}+\mathrm{Na}]^{+}$ion & $m / z$ calculated for the $[\mathrm{M}+\mathrm{Na}]^{+}$ion \\
\hline FucGal $_{2}$-nLNO & 1930.73 & 1930.67 \\
\hline Fuc-nLND & 1971.73 & 1971.69 \\
\hline Gal-nLND & 1987.74 & 1987.69 \\
\hline $\mathrm{Fuc}_{2}$-nLND & 2117.81 & 2117.75 \\
\hline FucGal-nLND & 2133.81 & 2133.75 \\
\hline $\mathrm{Gal}_{2}-\mathrm{nLND}$ & 2149.81 & 2149.74 \\
\hline \multirow[t]{2}{*}{$\mathrm{NeuAcFuc}_{3}-\mathrm{nLNO}$} & 2189.78 & 2189.77 \\
\hline & $2211.80[\mathrm{M}-\mathrm{H}+2 \mathrm{Na}]^{+}$ & 2211.75 \\
\hline $\mathrm{NeuAcFuc}_{2}$ Gal-nLNO & 2205.89 & 2205.77 \\
\hline NeuAcFuc-nLND & 2262.81 & 2262.78 \\
\hline $\mathrm{Fuc}_{3}-\mathrm{nLND}$ & 2263.89 & 2263.81 \\
\hline NeuAcGal-nLND & 2278.85 & 2278.78 \\
\hline $\mathrm{Fuc}_{2} \mathrm{Gal}-\mathrm{nLND}$ & 2279.93 & 2279.80 \\
\hline FucGal $_{2}-\mathrm{nLND}$ & 2295.91 & 2295.80 \\
\hline $\mathrm{Gal}_{3}-\mathrm{nLND}$ & 2311.85 & 2311.79 \\
\hline \multirow{2}{*}{$\mathrm{NeuAcFuc}_{2}-\mathrm{nLND}$} & 2408.91 & 2408.85 \\
\hline & $2430.86[\mathrm{M}-\mathrm{H}+2 \mathrm{Na}]^{+}$ & 2430.83 \\
\hline $\mathrm{Fuc}_{4}-\mathrm{nLND}$ & 2409.97 & 2409.87 \\
\hline \multirow[t]{2}{*}{ NeuAcFucGal-nLND } & 2424.88 & 2424.84 \\
\hline & $2446.93[\mathrm{M}-\mathrm{H}+2 \mathrm{Na}]^{+}$ & 2446.82 \\
\hline $\mathrm{Fuc}_{3} \mathrm{Gal}-\mathrm{nLND}$ & 2425.89 & 2425.86 \\
\hline $\mathrm{NeuAcGal}_{2}-\mathrm{nLND}$ & 2440.84 & 2440.84 \\
\hline $\mathrm{Fuc}_{2} \mathrm{GaI}_{2}-\mathrm{nLND}$ & 2441.90 & 2441.86 \\
\hline $\mathrm{FucGal}_{3}$-nLND & 2457.89 & 2425.86 \\
\hline $\mathrm{NeuAc}_{2}$ Fuc-nLND & 2553.89 & 2553.88 \\
\hline $\mathrm{NeuAcFuc}_{3}-\mathrm{nLND}$ & 2554.92 & 2554.90 \\
\hline $\mathrm{NeuAc}_{2} \mathrm{Gal}^{\mathrm{J}} \mathrm{nLN}$ & 2569.90 & 2569.88 \\
\hline $\mathrm{NeuAcFuc}_{2} \mathrm{Gal}_{-n L N D}$ & 2570.99 & 2570.90 \\
\hline $\mathrm{NeuAcFucGal}_{2}$-nLND & 2586.95 & 2586.89 \\
\hline NeuAcFuc-nLNDD & 2628.04 & 2627.92 \\
\hline NeuAcGal-nLNDD & 2643.97 & 2643.92 \\
\hline $\mathrm{NeuAc}_{2} \mathrm{Fuc}_{2}$-nLND & 2700.00 & 2699.94 \\
\hline $\mathrm{NeuAcFuc}_{4}$-nLND & 2701.03 & 2700.96 \\
\hline $\mathrm{NeuAc}_{2}$ FucGal-nLND & 2715.96 & 2715.94 \\
\hline $\mathrm{NeuAcFuc}_{2}$-nLNDD & 2774.05 & 2773.98 \\
\hline NeuAcFucGal-nLNDD & 2790.05 & 2789.97 \\
\hline $\mathrm{NeuAc}_{2} \mathrm{Fuc}_{3}-\mathrm{nLND}$ & 2846.14 & 2846.00 \\
\hline $\mathrm{NeuAcFuc}_{2} \mathrm{Gal}_{-n L N D D}$ & 2936.10 & 2936.03 \\
\hline $\mathrm{NeuAcFucGal}_{2}-\mathrm{nLDD}$ & 2952.01 & 2952.03 \\
\hline $\mathrm{NeuAcFuc}_{2}-\mathrm{nLNTD}$ & 3139.18 & 3139.11 \\
\hline NeuAcFucGal-nLNTD & 3155.14 & 3155.10 \\
\hline $\mathrm{NeuAcGal}_{2}-\mathrm{nLNTD}$ & 3171.14 & 3171.10 \\
\hline $\mathrm{NeuAcFuc}_{2} \mathrm{Gal}_{-n L N T D}$ & 3301.12 & 3301.16 \\
\hline $\mathrm{NeuAcFucGal}_{2}-\mathrm{nLNTD}$ & 3317.22 & 3317.16 \\
\hline
\end{tabular}

though not available on the used instrument, is expected to be straightforwardly achievable.

\section{Acknowledgments}

The authors thank Sequenom GmbH (Hamburg, Germany) for use of their o-TOF-instrument, Michael Flügger for providing the elephant milk sample, Johannes Müthing for use of HPTLC equipment, Andreas Rohlfing for technical assistance, and Franz Hillenkamp for support of the project. The authors gratefully acknowledge financial support by the Deutsche Forschungsgemeinschaft under grant DR416/5-1.

\section{References}

1. Holmes, E. W.; O’Brien, J. S. Separation of glycoprotein-derived oligosaccharides by thin-layer chromatography. Anal. Biochem. 1979, 93, $167-170$.
2. Kunz, C.; Rudloff, S.; Schad, W.; Braun, D. Lactose-derived oligosaccharides in the milk of elephants: Comparison with human milk. Br. J. Nutr. 1999, 82, 391-399.

3. Fenn, J. B.; Mann, M.; Meng, C. K.; Wong, S. F.; Whitehouse, C. M. Electrospray ionization for mass-spectrometry of large biomolecules. Science 1989, 246, 64-71.

4. Karas, M.; Hillenkamp, F. Laser Desorption ionization of proteins with molecular masses exceeding 10,000 Daltons. Anal. Chem. 1988, 60, 2299-2301.

5. Stahl, B.; Steup, M.; Karas, M.; Hillenkamp, F. Analysis of neutral oligosaccharides by matrix-assisted laser desorption ionization mass spectrometry. Anal. Chem. 1991, 63, 1463-1466.

6. Carr, S. A.; Huddleston, M. J.; Bean, M. F. Selective identification and differentiation of N-linked and O-linked oligosaccharides in glycoproteins by liquid chromatography mass spectrometry. Protein Sci. 1993, 2, 183-196.

7. Bahr, U.; Karas, M. Differentiation of "isobaric" peptides and human milk oligosaccharides by exact mass measurements using electrospray ionization orthogonal time-of-flight analysis. Rapid Commun. Mass Spectrom. 1999, 13, 1052-1058.

8. Reinhold, V. N.; Reinhold, B. B.; Costello, C. E. Carbohydrate molecular weight profiling sequence, linkage, and branching data ES-MS and CID. Anal. Chem. 1995, 67, 1772-1784. 
9. Wilson, I. D. The state of the art in thin-layer chromatography-mass spectrometry: A critical appraisal. J. Chromatogr. A 1999, 856, 429-442.

10. Gusev, A. I. Interfacing matrix-assisted laser desorption/ionization mass spectrometry with column and planar separations. Fresenius J. Anal. Chem. 2000, 366, 691-700.

11. Ivleva, V. B.; Elkin, Y. N.; Budnik, B. A.; Moyer, S. C.; O'Connor, P. B.; Costello, C. E. Coupling thin-layer chromatography with vibrational cooling matrix-assisted laser desorption/ionization Fourier transform mass spectrometry for the analysis of ganglioside mixtures. Anal. Chem. 2004, 76, 6484-6491.

12. Dreisewerd, K.; Müthing, J.; Rohlfing, A.; Meisen, I.; Vukelic, Z.; Peter-Katalinic, J.; Hillenkamp, F.; Berkenkamp, F. Analysis of gangliosides directly from thin-layer chromatography plates by infrared matrix-assisted laser desorption/ionization orthogonal time-of-flight mass spectrometry with a glycerol matrix. Anal. Chem. 2005, 77, 4098-4107.

13. Guittard, J.; Hronowski, X. P. L.; Costello, C. E. Direct matrix-assisted laser desorption/ionization mass spectrometric analysis of glycosphingolipids on thin layer chromatographic plates and transfer membranes. Rapid Commun. Mass Spectrom. 1999, 13, 1838-1849.

14. Ford, M. J.; Van Berkel, G. J. An improved thin-layer chromatography/ mass spectrometry coupling using a surface sampling probe electrospray ion trap system. Rapid Commun. Mass Spectrom. 2004, 18, 13031309 .

15. Luftmann, H. A simple device for the extraction of TLC spots: Direct coupling with an electrospray mass spectrometer. Anal. Bioanal. Chem. 2004, 378, 964-968

16. O'Connor, P.; Budnik, B.; Ivleva, V.; Kaur, P.; Moyer, S. C.; Pittman, J. L.; Costello, C. E. A. high pressure matrix-assisted laser desorption ion source for Fourier transform mass spectrometry designed to accommodate large targets with diverse surfaces. J. Am. Soc. Mass Spectrom. 2004, $15,128-132$

17. Hayen, H.; Volmer, D. A. Rapid identification of siderophores by combined thin-layer chromatography/matrix-assisted laser desorption/ionization mass spectrometry. Rapid Commun. Mass Spectrom. 2005, $19,711-720$.

18. Loboda, A. V.; Ackloo, S.; Chernushevich, I. V. A. high-performance matrix-assisted laser desorption/ionization orthogonal time-of-flight mass spectrometer with collisional cooling. Rapid Commun. Mass Spectrom. 2003, 17, 2508-2516.

19. Dreisewerd, K. The desorption process in MALDI. Chem. Rev. 2003, 103, 395-425.

20. Chang, T. T.; Lay, J. O.; Francel, R. J. Direct analysis of thin-layer chromatography spots by fast atom bombardment mass spectrometry. Anal. Chem. 1984, 56, 109-111.
21. Karlsson, K. A.; Lanne, B.; Pimlott, W.; Teneber, S. The resolution into molecular species on desorption of glycolipids from thin-layer chromatograms, using combined thin-layer chromatography and fast-atombombardment mass spectrometry. Carbohydr. Res. 1991, 221, 49-61.

22. Dreisewerd, K.; Berkenkamp, S.; Leisner, A.; Rohlfing, A.; Menzel, C. Fundamentals of matrix-assisted laser desorption/ionization mass spectrometry with pulsed infrared lasers. Int. J. Mass Spectrom. 2003, 226, 189-209.

23. Von Seggern, C. E.; Moyer, S. C.; Cotter, R. E. Liquid infrared atmospheric pressure matrix-assisted laser desorption/ionization ion trap mass spectrometry of sialylated carbohydrates. Anal. Chem. 2003, 75, 3212-3218.

24. Kunz, C.; Rudloff, S.; Baier, W.; Klein, N.; Strobel, S. Oligosaccharides in human milk: Structural, functional, and metabolic aspects. Annu. Rev. Nutr. 2000, 20, 699-722.

25. Chaturvedi, P.; Warren, C. D.; Altaye, M.; Morrow, A. L.; Ruiz-Palacios, G.; Pickering, L. K.; Newburg, D. S. Fucosylated human milk oligosaccharides vary between individuals and over the course of lactation. Glycobiology 2001, 11, 365-372.

26. Spina, E.; Cozzolino, R.; Ryan, E.; Garozzo, D. Sequencing of oligosaccharides by collision-induced dissociation matrix-assisted laser desorption/ionization mass spectrometry. J. Mass Spectrom. 2000, 35, 10421048.

27. Chai, W. G.; Piskarev, V.; Lawson, A. M. Negative ion electrospray mass spectrometry of neutral underivatized oligosaccharides. Anal. Chem. 2001, 73, 651-667.

28. Urashima, T.; Saito, T.; Nakamura, T.; Messer, M. Oligosaccharides of milk and colostrum in nonhuman mammals. Glycoconj. J. 2001, 18, 357-371.

29. Bruntz, R.; Dabrowski, U.; Dabrowski, J.; Ebersold, A.; Peter-Katalinic, J.; Egge, H. Fucose-containing oligosaccharides from human milk from a donor of blood group-0 LEA nonsecretor. Biol. Chem. Hoppe-Seyler 1988, 369, 257-273.

30. Svennerholm. L. The quantitative estimation of cerebrosides in nervous tissue. J. Neurochem. 1956, 1, 42-53.

31. Cramer, R.; Corless, S. Liquid ultraviolet matrix-assisted laser desorption/ionization mass spectrometry for automated proteomic analysis. Proteomics 2005, 5, 360-370.

32. Ivleva, V. B.; Sapp, L. M.; O'Connor, P. B.; Costello, C. E. Ganglioside analysis by thin-layer chromatography matrix-assisted laser desorption/ionization orthogonal time-of-flight mass spectrometry. J. Am. Soc. Mass. Spectrom. 2005, 16, 1552-1560.

33. Sze, E. T. P.; Chan, T. W. D.; Wang, G. Formulation of matrix solutions for use in matrix-assisted laser desorption ionization of biomolecules. J. Am. Soc. Mass Spectrom. 1998, 9, 166-174. 